Dorothea Schmidt

\title{
Geschlossene Gesellschaft? Die säkulare Entwicklung der Konzentration in der deutschen Elektroindustrie
}

\section{Zwang zur Größe: nicht erst ein Schlachtruf unserer Tage}

Die konjunkturelle Krise, die das 20. Jahrhundert in Deutschland einläutete, traf die Elektroindustrie, seit den bewegten Gründerjahren fast stetig im Aufwind, besonders hart. Mehr noch als in allen anderen Branchen schienen hier Großunternehmen bereits das Geschehen zu dominieren, und doch wiesen auch einige von ihnen deutliche Anzeichen von Schwäche auf. Es kam zu Kapitalabwertungen, zur Aussetzung von Dividendenzahlungen und zu Konkursen. Nur die beiden größten Einzelkapitale, Siemens \& Halske und die AEG, überstanden den ökonomischen Wirbelsturm einigermaßen unbeschadet und begannen unverzüglich, sich die Überreste ihrer teils zerzausten, teils zerstörten Konkurrenten einzuverleiben. Die AEG übernahm in der Folge unter anderem die Union, Siemens \& Halske unter anderem die Nürnberger Firma Schuckert (Pohl 1978: 12f). Emil Rathenau, als Gründer und Leiter der AEG einer der maßgeblich Beteiligten bei diesen Prozessen, zeigte sich überzeugt davon, die hiermit entstandene Situation werde sich als allseits segensreich erweisen:

»Die gegenwärtige Lage der Industrie macht den Zusammenschluß der elektrotechnischen Firmen zu einer Notwendigkeit. Die wirtschaftlichen Vorteile des Zusammenschlusses sind so erheblich, daß ihnen gegenüber alle Bedenken verschwinden. Interessen dritter werden nicht verletzt: weder einzelner noch der Allgemeinheit. Dem Lande aber wird das Fortbestehen einer seiner schönsten und stärksten Industrien gesichert« (zit. bei Loewe 1903: 143)

Auch im Geschäftsbericht der Deutschen Bank fand sich eine ähnlich enthusiastische Einschätzung, ging man ja ohnehin davon aus, der »Zug der Zeit« dränge »unwiderstehlich zur Bildung machtvoller Einheiten« (zit. bei Kreller 1903: 26, FN).

Zeitgenossen, die den Geschehnissen etwas ferner standen, sahen diese zwar weitaus weniger positiv, hielten den anhaltenden Trend zur Konzentration des Kapitals jedoch angesichts der »verwüstenden « Preiskonkurrenz, die man jüngst erlebt hatte, für ebenso unabwendbar. So fragte sich der Abgeordnete der Fortschrittlichen Volkspartei Oeser in einer Reichstagssit- 
zung, »ob nicht morgen schon der große elektrische Trust fertig abgeschlossen ist, der die gesamten deutschen Großunternehmungen in eine Hand bringt, sie unter einen einheitlichen Willen zwingt« (Verhandlungen d. Reichstags, 16.3.1911: 5527, 5530) Ähnlich prognostizierte der Berichterstatter des Vereins für Socialpolitik:

»Ist aber einmal die gesamte elektrotechnische Großindustrie in zwei grosse Gruppen vereinigt, so ist bis zur Bildung eines einheitlichen deutschen Elektrizitätstrusts nur noch ein kleiner Schritt. Ist doch heute schon die Konkurrenz zwischen den beiden grossen Gruppen, die in Berlin zusammensitzen, trotz aller äussern Gegensätze mehr nur noch eine scheinbare." (Kreller 1903: 28)

Andere Beobachter der Zeitläufte gingen in ihrer Einschätzung des neuen Phänomens noch sehr viel weiter. Lenin etwa vertrat in seiner populären Schrift Der Imperialismus als höchstes Stadium des Kapitalismus, die Fusionen und Aufkäufe der deutschen Elektroindustrie stellten in Kombination mit den Verträgen, die diese mit der amerikanischen General Electric abgeschlossen hatte, überhaupt das Modell der künftigen Entwicklung des Kapitals dar:

»Wir sehen hier anschaulich, wie sich in der Epoche des Finanzkapitals private und staatliche Monopole miteinander verflechten und die einen wie die anderen in Wirklichkeit bloß einzelne Glieder in der Kette des imperialistischen Kampfes zwischen den größten Monopolisten um die Teilung der Welt sind « (Lenin 1917: 83-4).

Angesichts der neuen Welle von Fusionen, die seit einigen Jahren nicht nur die Elektroindustrie erfaßt hat, ist aus dem Munde der jetzigen Hauptakteure der gleiche Tenor von der Zwangsläufigkeit zu hören, die ihr Handeln leite und ihnen keine Alternative ließe - nur die Begründungen klingen mittlerweile anders. Der Siemens-Konzern etwa hat seit den achtziger Jahren eine Reihe von spektakulären Firmenaufkäufen getätigt: unter anderem die US-amerikanischen Unternehmen Rolm (Telefone) und Arco (Solarzellen), Teile der britischen Plessey-Gruppe (Telekommunikation), die Mehrheit der deutschen Firma Nixdorf (Computer) sowie das ebenfalls deutsche Unternehmen für Schienenfahrzeuge Düwag AG (Welzmüller 1991: 623). Für den Vorstandsvorsitzenden Heinrich v. Pierer tragen diese Übernahmen dazu bei, sowohl die Ansprüche der 600.000 Aktionäre zu befriedigen, wie auch mit den 200.000 deutschen Beschäftigten »verantwortungsvoll« umzugehen:

»Wir gehen systematisch und bedacht vor. Wir verstärken unser Portfolio, um in führende Weltmarktpositionen zu kommen. Aber das wird uns nicht überall aus eigener Kraft gelingen. Dann müssen wir zukaufen - siehe den Erwerb des Geschäfts mit Briefsortieranlagen von Daimler-AEG -, Kooperationen eingehen oder im Extremfall auch mal zurückstecken « (Wirtschaftswoche, Nr. 8/1997: 42).

Von Emil Rathenau zu Heinrich v. Pierer: Was sich am Beginn des 20. Jahrhunderts dem Erschrecken vor den Folgen ruinöser Konkurrenz auf nationaler Ebene verdankte, wird am Ende desselben Jahrhunderts mit der 
Furcht vor dem Zurückbleiben im internationalen Wettbewerb gerechtfertigt. Dies wirft die Frage nach dem Stellenwert dieser Entwicklungen auf: Stehen wir vor einer Erscheinung des Wiedergängertums? Ist der Dämon, der die kleineren Anbieter ins Verderben trieb und den Giganten großzügig die profitable Nutzung der Märkte überließ, damals erstmalig aufgetaucht, um dann zu verschwinden und nun abermals auf den Plan zu treten? Oder handelt es sich einfach um die lineare Fortsetzung eines unumkehrbaren Prozesses, der einmal begonnen, zwangsläufig weitergehen muß, wie etwa von der Arbeitsgruppe Alternative Wirtschaftspolitik angenommen wird, wenn sie die »Existenz von Konzernen und Konzerngruppen« diagnostiziert, »die seit hundert Jahren die vorderen Plätze in der Wirtschaft belegen und sich als wirtschaftliche und politische Machtfaktoren etabliert haben« (1988: 11). Diese Autoren formulieren das auch sonst weitverbreitete Verständnis, es gebe eine langfristig kontinuierliche Zunahme der Konzentration, die in Deutschland allenfalls kurzzeitig durch politische Interventionen wie die nach 1945 verordneten Entflechtungen unterbrochen worden sein soll - eine Vorstellung, wonach mit der Durchsetzung marktwirtschaftlicher Verhältnisse auch simultan ein Mechanismus der stetig zunehmenden Zusammenballung von Kapital in Gang gesetzt worden sei.

Doch gerade Marx, der als Ahnherr dieser Behauptung gilt, betonte die Widersprüchlichkeit der Prozesse von Konzentration (Wachstum der individuellen Kapitale) und Zentralisation (Zusammenfassung vorher selbständiger Kapitale). »Je ein Kapitalist schlägt viele tot«: Damit beschwor Marx zwar ein drastisches Bild der Vorgänge, bei denen Existenzen und Lebensentwürfe kleinerer Unternehmer zerstört wurden, aber diese eingängige und später besonders gern zitierte Formulierung hinderte Marx nicht daran, auch mögliche Gegentendenzen der beschriebenen Prozesse ins Auge zu fassen (Marx 1867: 790). Der Attraktion der Kapitale stehe ihre Repulsion gegenüber, der Tendenz zur Zusammenballung eine solche der Zersplitterung: $» \ldots$ das Wachstum der funktionierenden Kapitale ist durchkreuzt durch die Bildung neuer und die Spaltung alter Kapitale« (ebd.: 654). Auch gebe es immer wieder Nischen:

»Die kleineren Kapitale drängen sich daher in Produktionssphären, deren sich die große Industrie nur noch sporadisch oder unvollkommen bemächtigt hat. Die Konkurrenz rast hier im direkten Verhältnis zur Größe der rivalisierenden Kapitale« (ebd.: 655).

Das für den Kapitalismus zentrale Element der Konkurrenz bewirke also, daß die großen Kapitale nicht in der Lage sind, in aller Ungestörtheit ihre Machtposition auszukosten, da eine solche immer wieder gefährdet werden kann. Im Anschluß an diese Überlegungen ist im folgenden am Paradebeispiel der Elektroindustrie zu untersuchen, ob der Weg von Unternehmen, die schon vor einem Jahrhundert zu den großen gehörten, tatsächlich so geradlinig in Richtung einer zunehmend »geschlossenen Gesellschaft« ver- 
lief, wie es oftmals vermutet wird. Sind die Großen von gestern denn wirklich identisch mit den Großen von heute? Und wie kann, was vor dem Ersten Weltkrieg bereits fast an einem Endpunkt angekommen zu sein schien, später überhaupt noch weitere Steigerungen erfahren haben?

\section{Bestandsveränderungen: die Zahl der Betriebe und Unternehmen}

Einen ersten Anhaltspunkt, um die Entwicklung der Konzentration einzuschätzen, liefert die Veränderung der Zahl der Betriebe (als technische Einheiten) und Unternehmen (als wirtschaftliche Einheiten) innerhalb der Branche (wobei man sich dem Problem gegenübersieht, daß die vorhandenen Daten sich mal auf die eine, mal auf die andere Größe beziehen). Nach der Statistik des Deutschen Reichs betrug die Zahl der Betriebe, die sich auf dem Gebiet der Herstellung von Lampen- und Beleuchtungsapparaturen sowie von »elektrischen Maschinen, Apparaturen usw.« betätigten, im Jahr 1875 bereits 81, wobei selbst bei den schon länger bestehenden Firmen wie Siemens \& Halske oder Schuckert Betrieb und Unternehmen in den ersten Jahrzehnten praktisch zusammenfielen. So war der Geschäftsbetrieb von Siemens \& Halske 1847 mit der Herstellung von Telegraphen aufgenommen worden und erstreckte sich in den folgenden Jahren auf eine mehr und mehr ausufernde Palette von elektrotechnischen Produkten aller Art. Diese weitgefächerte Produktion fand bis nach der Jahrhundertwende in einem einzigen zusammengeschachtelten Gebäudekomplex in der Berliner Markgrafenstraße statt. Die ursprüngliche Dominanz der Schwachstromtechnik wurde seit den siebziger Jahren durch die wachsende Bedeutung von Starkstromprodukten abgelöst. Die Entwicklung dieses Zweiges verdankte sich vor allem dem von Werner Siemens entdeckten elektro-dynamischen Prinzip der Selbsterregung des Magnetfeldes, auf dessen Grundlage es ebenso möglich war, elektrische aus mechanischer Energie zu erzeugen, wie umgekehrt mechanische Energie in elektrische zu verwandeln. Beides erschloß eine Fülle von neuen Anwendungen in der Industrie und im Verkehrswesen. Bis Mitte der neunziger Jahre nahm die Zahl der Betriebe auf mehr als $1.000 \mathrm{zu}$, und 1907 gab es schon fast 4.000 elektrotechnische Werkstätten und Fabriken (Wellhöner 1989: 214, Statistik des Deutschen Reiches, Bd. 220/21: 74*ff).

Einer der Gründe für diese rasante Zunahme der Zahl von Betriebsstätten lag vor allem seit der Jahrhundertwende darin, daß die etablierten Firmen sich vergrößerten, indem sie für die neuen Produktionszweige eigene Werke erbauten. Siemens etwa, mittlerweile am Berliner Nonnendamm angesiedelt, errichtete in der späteren Siemensstadt unter anderem spezielle Kabel-, Dynamo- und Kleinbauwerke. Die AEG, Emil Rathenaus Gründung aus den achtziger Jahren und ebenso wie Siemens als Universalunterneh- 
men in fast allen Sparten der Elektrotechnik engagiert, expandierte über das ursprüngliche Stammwerk in der Berliner Ackerstraße hinaus und legte sich weitere Fabrikationseinrichtungen in der nahen Brunnenstraße zu. Aber die Vermehrung der Betriebe ging darüber hinaus auch auf eine bewegte Gründungsdynamik zurück. In der Wahrnehmung der Zeitgenossen schossen elektrotechnische Werkstätten damals »wie Pilze aus dem Boden« (Loewe 1903: 112), was unter anderem die Entstehung von Spezialfabriken zur Folge hatte. Sie begannen meist als Eigentümer-Unternehmen und waren nach heutigen Begriffen der mittelständischen Wirtschaft zuzurechnen, wie die Elektrizitätszählerfabrik G. Aron in Charlottenburg oder die Deutsche Glühlicht AG Auergesellschaft und die Telephon- und Telegraphenwerke Mix \& Genest in Berlin-Schöneberg (Müller 1925: 49). Nach dem Urteil eines kundigen Beobachters der Branche waren sie imstande, »bei ausgezeichneter Ausnützung der Massenfabrikation gegenüber den Grossfirmen mit sehr kleinem und billigem Verwaltungsapparat auskommen zu können. Sie sind diesen gegenüber meist sogar sehr konkurrenzfähig « (Kreller 1903: 9). Bestimmte Produktionsgruppen wie etwa die elektrischen Kraftzentralen teilten die großen Firmen unter sich auf, aber Bereiche mit geringerem Kapitalaufwand stellten für die kleinen und mittelgroßen Unternehmen keineswegs unerhebliche Nischen dar. So fertigten sie unter anderem Meßinstrumente, Telefonapparate, Zähler, Bogenlampen, Schaltapparate, Isoliermaterialien, Kohlenelektroden, Elektromotoren, Glühlicht und Akkumulatoren. Manche trieben die Spezialisierung noch weiter, indem sie beispielsweise nur Bogenlampen für lange Brenndauer oder nur Anlaßapparate für Motoren fabrizierten. Eine ganze Reihe dieser Spezialfabriken erreichte vor dem Ersten Weltkrieg solche Dimensionen, daß sie sich als Aktiengesellschaften etablieren konnten, darunter etwa die Telefon- und Telegrafenfirma Mix \& Genest oder die Welter Elektrizitäts- und Hebezeugwerk AG Köln (Kreller 1903: 9, Müller 1925: 49ff, Loewe 1903: 103ff). Eine andere, häufig ebenfalls erfolgreiche Strategie verfolgten Werkstätten, die sich auf den lokalen und regionalen Märkten behaupteten, und dabei - im Prinzip nicht anders als die großen Universalfirmen - fast die gesamte Palette der Schwachstrom- und Starkstromelektrik abdeckten. Viele von ihnen begannen als Ein-Mann-Betriebe - wie Alois Zettler, ein gelernter Feinmechaniker, der 1877 in München eine Werkstatt eröffnete, in der er zunächst so unterschiedliche Produkte wie Theodolite, Nivellierinstrumente, Kippregler und Distanzmesser, Rühmkorffsche Induktionsapparate, Elektrisier- und Influenzmaschinen oder Galvanoskope herstellte. Bald konnte er den Betrieb erweitern und erhielt aufgrund seiner Teilnahme an der Münchner Elektrizitätsausstellung von 1882 den Auftrag für die Illumination des Hoftheaters, installierte Beleuchtungen für die Kesselschmiede Maffei sowie die Münchnerkindl-Brauerei und eine Schloßbe- 
leuchtung in Herrenchiemsee, worauf er er den Titel »Erster Hofilluminator « erhielt. Die wachsende Zahl der Aufträge erlaubten ihm, seine Firma, die er nun als »Elektrotechnische Fabrik« bezeichnete, auf 80 Beschäftigte zu vergrößern (Zettler o.J.: 7ff, 45).

Im Zug der wirtschaftlichen Aufbruchsstimmung nach dem Ende der »großen Depression « der achtziger und neunziger Jahre führten die genannten Erweiterungen und Neugründungen zu Überkapazitäten, die in einer Berliner Zeitung damals folgendermaßen kommentiert wurden:

»Neigen selbst Industrien, die schon alte Erfahrungen für sich haben, immer wieder zu derartigen Überschätzungen hin, so sprudelte die Elektrizität mit dem Ungestüm der Jugend über. Die großen Gesellschaften dehnten sich aus, als ob jede allein für sich die Welt mit Elektrizität zu versorgen hätte« (zit. bei Loewe 1903: 112).

Es kam zu den eingangs erwähnten krisenhaften Zusammenbrüchen nach der Jahrhundertwende, die sich vorübergehend dämpfend auf die bisherige Expansion auswirkten. Bald aber setzte ein neues Gründungsfieber ein. Im Jahrzehnt vor dem Ersten Weltkrieg stieg nicht nur die Zahl der mittelgroßen Spezialfirmen wieder an, selbst die Zahl der kleinen und kleinsten Betriebe, also solche mit weniger als 5 Beschäftigten nahm zu (Statistik des Deutschen Reiches, Bd. 220/21: 33*). Sie alle wollten, wie Alois Zettler, »an dem endgültigen Siege der Kultur über alle Kräfte der Finsternis « mitarbeiten (Zettler o.J.: 61) - oder vielleicht auch lediglich am erhofften Geldsegen einer Wachstumsbranche teilhaben.

Das Muster eines äußerst heterogenen Nebeneinanders von großen, mittleren und kleinen Firmen sollte sich in den folgenden Jahrzehnten und insbesondere in der Zwischenkriegszeit weiter fortsetzen. Trotz der Dominanz der großen Unternehmen hielt sowohl in den zwanziger wie in den dreißiger Jahren die Welle der Neugründungen an. Von 1907 bis 1925 nahm die Zahl der Betriebe in der Elektroindustrie von rd. 6.500 auf rd. 22.000, also um mehr als das Dreifache, zu, wobei die großen Firmen ihre Produktionsstätten kaum noch vermehrten, so daß der Zuwachs im großen und ganzen tatsächlich auf eine größere Zahl von Unternehmen zurückgeht (Fischer 1976: 809).

Die Gründungen fanden vor allem in den Zweigen statt, die sich in den zwanziger Jahren durch die Expansion der Elektrotechnik in den Konsumbereich eröffneten. Die bisherigen Branchenführer, Siemens und die AEG, blieben ihrer vorrangigen Investitionsgüterorientierung treu und befaßten sich allenfalls nebenbei mit den neuen Konsumgütern. Das wichtigste davon war das Radio. Zwar verfügten die beiden Unternehmen über ihre gemeinsame Tochter Telefunken mit den Lieben-Rechten quasi über ein Patentmonopol, doch mußten sie dieses unter dem Druck der Reichspost teilweise aufgeben und gegen Erstattung einer Gebühr Bauerlaubnisse für Radioapparate erteilen. Wenn die Zahl der angemeldeten Rundfunkteilnehmer 
bis 1933 um das Zehntausendfache und diejenige der produzierten Röhren in der kurzen Zeitspanne von 1923 bis 1931 von 102.125 auf 5,875 Mio stiegen, so ging dies vor allem auf Tausende privater Radiobastler, aber auch auf eine Unzahl neu gegründeter Firmen zurück, während der Marktanteil von AEG und Siemens \& Halske sowie der von ihnen kontrollierten Firmen bei 1/3 blieb (Czada 1969: 279). Viele der Newcomer konnten sich jedoch auf die Dauer nicht halten, so daß am Ende des Jahrzehnts nur noch etwa 300 übrig blieben, darunter die Firmen Saba, Mende und Braun (Petzold 1987: 347, Wittke 1996: 111f).

Insgesamt ging die Zahl der Unternehmen in der Elektrotechnik seit Mitte der zwanziger Jahre zurück, aber dieser Trend, der sich vor allem in der extrem schwierigen Periode nach 1929 verstärkte, kehrte sich ab 1933 einige Jahre lang um. Später allerdings fielen den »Auskämmungsaktionen« des nationalsozialistischen Regimes vor allem kleinere Firmen zum Opfer. Mit dem Zusammenbruch des Dritten Reiches fand auch diese politische Leitlinie ihr Ende, und die Periode des wirtschaftlichen Aufschwungs seit Beginn der fünfziger Jahre war - wie in früheren Zeiten - abermals von einer Welle von Neugründungen begleitet, die insbesondere seit den sechziger Jahren erheblich zunahmen. Belief sich der Bestand an Unternehmen 1936 auf rd. 1.500, so 1963 auf mehr als 2.000 und 1990 auf 3.000 - er hat sich somit in fünf Jahrzehnten verdoppelt. Die Schwerpunkte lagen dabei in einzelnen Jahrzehnten auf unterschiedlichen Produktionsgebieten: in den fünfziger und sechziger Jahren vor allem bei Haushaltsgeräten - hier ist an die Firmen Bauknecht, Miele, Zanker und Neff zu denken - und bei Unterhaltungselektronik - unter anderem Grundig, Metz, Becker und Tonfunk. Seit den 70er Jahren hat sich die Datenverarbeitungstechnik als besonders expansiv erwiesen (Czada 1969:94, Germann 1940: 39, Breitenacher 1974: 42, Berger 1993: 35, Wittke 1996: 132ff, Pohl 1978: 33).

Im Rahmen ihrer Studie Die kleine Fabrik trafen die Autoren Hilbert/ Sperling auf einige der typischen Neugründungen aus den siebziger Jahren. Fast immer sind es Techniker oder Ingenieure, die kürzere oder längere Zeit bei einem Großunternehmen der Branche tätig waren und sich dann als »technische Entrepreneure« selbständig machen, oftmals auf der Basis eigener technischer Entwicklungen - wie jener Ingenieur, der nach fast zwanzigjähriger Tätigkeit in einem großen Elektrokonzern ein eigenes kleines Unternehmen gründete, in dem er Patente verwertete, die er im Rahmen seiner Konstruktions- und Entwicklungsarbeit erworben hatte. Die großen Unternehmen fungieren in solchen Fällen als »Sprungbrett für Eigengründungen«, es handelt sich also um »split-off-Gründungen, bei denen die Idee der Gründung aus der Tätigkeit beim Inkubator resultiert, die Gründung selbst aber ohne Unterstützung und Einverständnis durch diesen erfolgt« (Hilbert/Sperling 1990: 84). Die neuen Firmen können sich als Zulieferer 
etablieren oder aber Marktnischen ausfüllen, die sich der gerade in den letzten Jahrzehnten stark zunehmenden Produktdifferenzierung verdanken. Beispielhaft dafür sind etwa ein Kleinbetrieb für Agrarelektronik, der »Bordcomputer « für Mähdrescher, Feldspritzen, Häcksler und Sähmaschinen fertigt, oder ein solcher für Leiterplatten, in dem hochwertige Produkte für die Industrieelektronik wie auch für die Luft- und Raumfahrtindustrie produziert werden (ebd.: 118f).

\section{Größenstrukturen: Beschäftigte}

Angaben über die Zahl von Betrieben und Unternehmen besagen noch nichts über deren Größe und Bedeutung als Arbeitgeber und als Anbieter auf bestimmten Märkten. Doch hat sich bisher immerhin gezeigt, daß es durch die bereits vor 1914 gegebene Dominanz großer Unternehmen zwar teilweise zum Niedergang mittlerer und kleinerer Unternehmen gekommen ist, solche aber auch immer wieder neu entstanden sind. Näheren Aufschluß verspricht die Betrachtung der Verteilung von Arbeitskräften und Umsätzen auf die einzelnen Betriebe und Unternehmen. In der Periode vor dem Ersten Weltkrieg wuchs die Zahl der Beschäftigten noch sehr viel stärker an als diejenige der Betriebe. Allein im Zeitraum von 1895 bis 1907 stieg sie von rd. 35.000 auf rd. 145.000, also um mehr als das Dreifache, wobei die Durchschnittsgröße der Betriebe genauso wie in anderen Branchen zunahm. In den drei Jahrzehnten, die auf Gründerboom und Gründerkrise folgten, von den achtziger Jahren bis zum Ersten Weltkrieg, reduzierte sich der Anteil (nicht jedoch die absolute Zahl) der Beschäftigten, die in Betrieben mit weniger als 50 Beschäftigten arbeiteten, in dieser Branche erheblich. Auch traten hier »Riesenfabriken« mit mehr als 1.000 Beschäftigten noch wesentlich stärker in Erscheinung als etwa im Bergbau oder in der Chemischen Industrie, ein Umstand, der neben anderen erklärt, warum die Zustände in dieser Branche von den Zeitgenossen als so besonders dramatisch angesehen wurden: Bei Siemens \& Halske belief sich die Belegschaftszahl um die Jahrhundertwende auf fast 14.000, bei der AEG lag sie sogar darüber (Loewe 1903: 90). In den folgenden Jahren waren mehr als $40 \%$ aller Arbeitskräfte in der Elektroindustrie in Betrieben mit mehr als 1.000 Beschäftigten tätig - man denke an Peter Behrens' berühmte Turbinenhalle der AEG oder an die überlieferten Bilder endloser Reihen von Arbeiterinnen und Arbeitern in riesigen Werkssälen wie in den damaligen Spulenwickeleien oder Schraubendrehereien (vgl. Rogge 1983: 148). Dies galt auch noch für die Zeit nach dem Ersten Weltkrieg, allerdings war um die Mitte der zwanziger Jahre damit auch bereits der Höhepunkt dieser Entwicklung erreicht, was unter anderem daran zu sehen ist, daß der Beschäftigtenanteil in den jeweils 20,30 und 50 größten Betrieben dieser 
Branche bis 1939 sank. Bis in die fünfziger Jahre nahm der Anteil der Beschäftigten in den Betrieben mit einer Belegschaft von mehr als 1.000 Personen stetig ab, und auch die gewaltige Ausdehnung der Produktion in der Zeit des »Wirtschaftswunders « ließ diese großbetriebliche Form niemals wieder so prägend werden wie in den zwanziger Jahren (Statistik des Deutschen Reichs, Bd. 220/21: 74*ff, Leicht 1995: 102f, Sombart 1928: 836, Pohl 1978: 40).

Bezog sich die bisherige Betrachtung auf die Ebene der Betriebsgrößen, so sind für die Zeit ab 1936 Daten verfügbar, die Aufschluß darüber geben, wie sich die Verteilung der Beschäftigten in der Elektroindustrie auf verschiedene Unternehmensgrößen verändert hat. Nach einer Statistik der Wirtschaftsgruppe Elektroindustrie entfielen 1936 auf lediglich 2,4\% der Firmen rd. 2/3 aller Beschäftigten. Dies waren die Unternehmen mit mehr als 1.000 Beschäftigten, worunter sich die großen Konzernunternehmen ebenso wie einige Spezialfabriken befanden. Das übrige Drittel der Beschäftigten war überwiegend in Firmen mit 200 bis 1.000, zu einem geringen Teil auch in Firmen mit weniger als 200 Beschäftigten tätig. Der Anteil der mittleren und kleineren Firmen dürfte sich in den folgenden Jahren angesichts der bereits erwähnten »Auskämmungsaktionen« des Regimes und aller sonstigen Bestrebungen, die Produktion auf größere Einheiten zu konzentrieren, weiter verringert haben (Germann 1940: 39, Siegel/von Freyberg 1991: 288f).

Nach dem Zweiten Weltkrieg führte die neuerliche Expansion der Branche dazu, daß die Arbeitsplätze Anfang der sechziger Jahre annähernd die Zahl von 1 Mio. erreichte, wobei sich trotz der erheblichen Ausweitung der Produktion und tiefgreifender Veränderungen ihrer Struktur (mit einem Bedeutungsverlust der konventionellen Elektrotechnik zugunsten der IndustrieElektronik) die Beschäftigung mit einigen Schwankungen bis 1990 auf diesem Niveau gehalten hat. Während der Beschäftigtenanteil der Firmen mit weniger als 200 Beschäftigten in den sechziger und siebziger Jahren auf 5\% gesunken war, ist er seither gestiegen, um 1990 mit 14\% einen ähnlichen Wert wie in den dreißiger Jahren zu erreichen; der Anteil der mittelständischen Firmen mit 200 bis 1.000 Beschäftigten schwankte in diesen Jahrzehnten immer um rd. 18\%, lag 1990 mit 20\% jedoch sogar höher als in den dreißiger Jahren. Der Anteil der Firmen mit mehr als 1.000 Beschäftigten stieg dagegen bis Ende der siebziger Jahre auf $77 \%$, ist seither aber auf $67 \%$ gesunken und hat sich somit auf das Niveau der dreißiger Jahre eingespielt. Dies hängt unter anderem damit zusammen, daß in den achtziger Jahren in den Großbetrieben Arbeitsplätze abgebaut, gerade in Kleinbetrieben aber neu entstanden sind (Welzmüller 1992: 32,Wittke 1996: 36, Breitenacher u.a 1974: 42, Berger 1993: 35, Cramer 1987: 27). Diese langfristigen Trends haben keineswegs zu einem Abbau der Dominanz der alt- 
eingesessenen Firmen geführt, dennoch zeigt sich eine gewisse Relativierung der bisherigen Entwicklung. Große Unternehmen haben fast von den Anfängen dieser Industrie an eine überragende Rolle für die Beschäftigung von Arbeitskräften gespielt: Für die Hälfte bis $2 / 3$ der in dieser Industrie Beschäftigten war der Erfahrungshorizont die Großindustrie. Doch hat sich deren Anteil in der Bundesrepublik nicht stetig vergrößert, sondern zeigt zumindest bis Ende der achtziger Jahre sogar eine leicht sinkende Tendenz.

\section{Größenstrukturen: Produktion und Umsätze}

Neben der Verteilung der Beschäftigten ist zur Einschätzung des Ausmaßes der Konzentration diejenige von Produktion und Umsätzen heranzuziehen. Die Angaben beziehen sich hier stets auf Firmen, weisen aber zumindest für die Vergangenheit gewisse Diskrepanzen auf. Häufig werden Zahlen angeführt, wonach die zwei größten Unternehmen des Kaiserreichs, Siemens und AEG, unmittelbar vor dem Ersten Weltkrieg rd. 65\% der Produktion auf sich vereinigten. Dem steht entgegen, daß nach anderen Schätzungen um 1911 von der jährlichen Gesamtproduktion in Höhe von 800 Mio Mark etwa 325 bis 350 Mio Mark, also mehr als 40\%, allein auf die Spezialfabriken entfielen, die auch einen erheblichen Anteil der Ausfuhr bestritten (Czada 1969: 279, Wittke 1996: 57, Verhandlungen d. Reichstags, 16.3.1911: 5528, Kreller 1903: 29).

Für die Zeit seit den zwanziger Jahren ergibt sich ein deutlicheres Bild. Offenbar erreichten die großen Konzerne - genauso wie bei ihrem Anteil an den Beschäftigten - in den zwanziger und dreißiger Jahren das Maximum ihres Anteils an Produktion und Umsätzen auf dem deutschen Markt. In dieser Zeit verschlechterte sich die Position der Spezialfabriken, wobei ihre Bedeutung bei einzelnen Produktionssparten sehr unterschiedlich blieb: bei Glühlampen und Leuchten wie bei Elektrokohlen erreichten sie nur Anteile unter 30\%, bei Maschinen, Apparaten und deren Zubehör knapp 40\%, bei Kabeln und Leitungen 50\%, bei Akkumulatoren, Batterien und Elementen aber 60\% (Germann 1940: 33, 40). Der Trend einer wachsenden Konzentration der wirtschaftlichen Aktivitäten auf die ganz großen Firmen fand in der Bundesrepublik keine Fortsetzung. So wird für die 25 größten Unternehmen des Jahres 1936 ein Anteil von 75\% angegeben, Anfang der siebziger und Ende der achtziger Jahre aber nur noch von $56 \%$ bzw. $57 \%$. Noch ausgeprägter ist diese Tendenz, wenn man die Betrachtung auf die allergrößten Unternehmen beschränkt. Entfielen auf die drei größten Unternehmen im Reichsgebiet von 1938 rd. 49\%, so im Bundesgebiet von 1951 rd. 36\%, 1970 41\%, $198032 \%$ und 1990 35\%. Ähnlich ging auch der Anteil der jeweils zwei größten Unternehmen zurück. 1936 kamen Siemens und die AEG zusammen auf 52\% der Umsätze, 1990 Siemens und Bosch 
jedoch nur noch auf rd. 36\% (Germann 1940: 35, Czada 1969: 279, Breitenacher u.a. 1974: 45, Berger 1984: 179, Berger 1993: 38, Bähr 1997: 76).

Aus den Daten zur Zahl der Betriebe und Unternehmen sowie zu deren Anteilen an Beschäftigung und Umsätzen ergibt sich, daß die Entwicklung seit der Jahrhundertwende sich nicht so geradlinig fortgesetzt hat, wie es damals allgemein erwartet worden war. Zwar weist allein Siemens in Deutschland heutzutage ebenso viele Beschäftigte auf, wie es sie 1913 in der gesamten deutschen Elektroindustrie gab, nämlich rd. 200.000 (dazu kommen noch etwa ebenso viele Arbeitskräfte in weiteren 190 Ländern) (Feldenkirchen 1988: 27, Lamparter 1997: 28). Dennoch war diese gewaltige Expansion nicht - wie Karl Kautsky 1891 in seinem Kommentar zum Erfurter Programm der Sozialdemokratie unheilvoll angekündigt hatte, vom »Todeskampf des Kleinbetriebs« begleitet. Es zeigte sich, daß die kleinen und mittleren Einheiten keineswegs Fossilien aus vorindustriellen Zeiten darstellten, die im Lauf der Zeit verwittern und verschwinden würden. Die Karten waren mit der Gründungsphase vor der Jahrhundertwende nämlich nicht - wie es den zeitgenössischen Beobachtern schien - ein für alle Mal verteilt worden, sondern es entwickelte sich auch hier der dynamische Prozeß einer periodischen Vernichtung und Neuentstehung von Kapitalen, auf den Marx das Augenmerk gelegt hatte. Neue Unternehmen profitierten vom Gesamtwachstum der Branche und von der Ausfächerung des Spektrums der elektrotechnischen Produkte, wobei manche von ihnen im Schatten der Großen und von diesen abhängig blieben, es anderen aber auch gelang, den Großunternehmen in gewissen Tätigkeitsbereichen Paroli zu bieten.

Deren Dominanz konnte somit immer wieder in Frage gestellt werden, so lange keine unüberwindlichen Eintrittsbarrieren für Neueinsteiger bestanden - und dies war bei den meisten elektrotechnischen Teilmärkten der Fall. Aber die starke Stellung großer Unternehmen sah sich nicht nur Gefährdungen von außen, sondern auch von innen ausgesetzt. Wiederholt hat man seit dem letzten Jahrhundert Erfolgsrezepte identifiziert, welche die überragende Position der Großen begründet und zementiert haben sollen: ihre bessere Finanzausstattung und ihr leichterer Zugang zum Kapitalmarkt, ihre Möglichkeiten für economies of scale und economies of scope, ihre Chancen der Diversifikation und der Risikoverteilung, ihre Stellung gegenüber Lieferanten und Kunden oder auch ihre oftmals privilegierten Beziehungen zu staatlichen Instanzen. Doch wissen wir mittlerweile, daß kaum einer dieser Faktoren langfristig eine Garantie für Expansion und Gewinne mit sich bringt. Was zeitweilig eine besondere Stärke begründet, kann unter anderen Umständen zu anhaltender Schwäche führen, wie einige spektakuläre Insolvenzen großer Unternehmen in den letzten Jahrzehnten gelehrt haben. Im folgenden wird diese Widersprüchlichkeit schlag- 
lichtartig für drei strategische Orientierungen dargestellt, die vor allem in der Elektroindustrie besonders zum Tragen gekommen sein sollen: 1) Forschung und Entwicklung, 2) Gründungsgeschäfte und Beteiligungen und 3) Kooperationen und Kartellbildungen. Dabei stehen die beiden Großfirmen im Mittelpunkt, die das Geschehen seit dem letzten Jahrhundert in erster Linie bestimmt haben: Siemens und die AEG.

\section{Dominanz durch Forschung und Entwicklung?}

Der Aufstieg der seit 1847 bestehenden Telegrafenfabrik von Siemens \& Halske hing zweifellos eng mit der Fülle technischer Innovationen Werner Siemens' selbst zusammen, dem das Erfinden und Weiterentwickeln Lebensbedürfnis war, und den es unendlich langweilte, sich mit ausgereiften und standardisierten Produkten zu befassen. Dem Rathenau-Biographen Riedler zufolge galt in den achtziger Jahren des 19. Jahrhunderts in der Branche als »allgemeiner Glaubenssatz, von niemand bestritten, alle Fragen von Bedeutung seien nur von Siemens zu lösen, niemand habe gleiche Einsicht und Erfahrung... Das berechtigte Ansehen der Person ging auch auf die Geschäfte über « (Riedler 1916: 56). Den wenigsten schien vorstellbar, daß dieses Unternehmen in absehbarer Zeit irgendwelche nennenswerte Konkurrenz erfahren könnte. Tatsächlich ist es Siemens in den folgenden Jahrzehnten in vielen Bereichen immer wieder gelungen, den Anspruch des Gründers einzulösen, sich stets »an der Spitze der Elektrotechnik zu halten « (Werner Siemens 1882 in einem Brief an seinen Bruder Karl, in: Matschoß [Hrsg.] 1916: 720-1). Aber häufig gingen solche Vorrangstellungen auch wieder verloren, unter anderem deshalb, weil leitende Mitarbeiter es angesichts des weitreichenden Renommees der Firma für überflüssig hielten, die konkreten Veränderungen von Märkten im Auge zu behalten. So wurde eine Reihe technischer Innovationen die Domäne von Spezialfabriken, insbesondere bei der Meßinstrumenten- und Röntgentechnik (Germann 1940: 26, FN, Voigt 1925: 19ff). Selbst kleinere Werkstätten konnten sich auf diesem Gebiet immer wieder profilieren. Der bereits erwähnte Münchner Alois Zettler etwa erfand eine Kontrollanlage für Nachtwächter und entwarf ein Patent für die elektrische Diebstahlsicherung von Ladenkassen und Tresoren (Zettler o.J.: 11). Und als in den achtziger Jahren des 19. Jahrhunderts unter den Elektrotechnikern eine mit religiösem Eifer betriebene Debatte über die Vor- und Nachteile von Gleichstrom und Wechselstrom ausbrach, war es die damals noch recht unbedeutende Firma Helios aus Köln, die sich dem geballten Sachverstand der etablierten Firmen entgegenstellte. Während Siemens, Schuckert und Lahmeyer 1891 bei der Frankfurter Elektrotechnischen Ausstellung antraten, um mit ihren Maschinen die Überlegenheit des herkömmlichen Gleichstromes zu demonstrie- 
ren, gelang den Konstrukteuren von Helios erstmals der praktische und für die Zukunft äußerst folgenreiche Nachweis, »daß hochgespannter Drehstrom auf große Entfernungen ohne übermäßigen Verlust geleitet und am Endpunkt auf Gebrauchsspannung herunter transformiert werden könne« (Voigt 1925: 17), so daß die in Frankfurt installierten Glühlampen zum Erstaunen der Besucher durch das 175 km entfernte Lauffener Kraftwerk illuminiert wurden.

Ähnliches wiederholte sich in den zwanziger Jahren, als die großen Unternehmen bei der Einführung des Rundfunks und der elektrischen Haushaltsgeräte überwiegend vornehme Zurückhaltung übten und diesen Bereich den neu auf den Markt strömenden Anbietern überließen. Volker Wittke betont in seiner Untersuchung über die Anfänge der Massenproduktion in der Elektrotechnik:

»Die eigentlichen Vorreiter der Konsumgüterproduktion in der Elektroindustrie waren demgegenüber Branchenneulinge. Bei Radios und bei Hausgeräten stellten die Startphasen massenhafter Verbreitung der neuen Produkte jeweils Zeiträume dar, in denen zahlreiche Neueinsteiger mit ausgesprochen vielfältigen Produktideen und Konzeptionen in den noch wenig konturierten Märkten auftauchten. Ob Radio, Kühlschrank und Waschmaschine: Vor allem Newcomer waren es, die der aufkeimenden Nachfrage mit den verschiedensten Formen technischer Umsetzung der Funktionsprinzipien Nahrung boten« (Wittke 1996: 109).

Auch in den letzten Jahrzehnten bestätigte sich mehrfach die mittlerweile gängige Erkenntnis, daß Höhe und Anteil der Ausgaben für Forschung und Entwicklung eines Unternehmens sich nicht zwangsläufig in dessen Innovationsfähigkeit niederschlagen. Ein erheblicher Aufwand in dieser Richtung kann ohne weiteres auch zu Produkt-Flops führen, und eine Unternehmenskultur mit weit zurückreichenden Traditionen sich geradezu lähmend auswirken. So gibt es bei Siemens seit den siebziger Jahren einen wachsenden Anteil von Mitarbeitern im Bereich Forschung und Entwicklung, und in der internationalen Patentstatistik beansprucht das Unternehmen einen der vorderen Plätze. Dennoch wird von Insidern auch die Kritik geäußert, es würden zu viel Mittel in die Perfektionierung alter Produkte gesteckt und man hielte unabhängig von den Anforderungen des Marktes hergebrachte und als »overengineered « geltende Siemens-Standards hoch. Beispielsweise gingen die von der Firma angebotenen Handys - »sündhaft teure, mit allerlei Schnickschnack vollgestopfte Geräte« (Der Spiegel Nr. 40/1998: 120) - an den Anforderungen des Marktes weitgehend vorbei. Auch beteilige man sich wenig an grundlegenden Neuerungen oder aber ließe solche, um angestammte Bereiche nicht zu gefährden, im Zweifelsfall in der Schublade verschwinden - Siemens nähme also mehr und mehr die »Position des schnellsten Verfolgers« ein (Welzmüller 1991: 625f, Fischer/Schwarzer 1997: 70, Naschold 1997: 10). In jüngster Zeit mehren sich allerdings auch die Meldungen, daß angesichts des Drucks der Konkurrenten häufig unausgereifte und mit erheblichen Qualitätsmängeln be- 
haftete Produkte die Werke verließen, wie etwa jüngst Dieselloks für die norwegischen Staatsbahnen, eine Müllverbrennungsanlage für die Stadt Fürth oder fehlerhafte Gasturbinen (Der Spiegel Nr. 40/1998: 118ff).

Angesichts dessen gelingt es auch heute kleineren und spezialisierteren Firmen immer noch, sich gegenüber den Giganten zu behaupten, beispielsweise Handwerksbetrieben bei der Antriebs-, Schalt- und Installationstechnik oder die - in der Tradition der alten Spezialfirmen stehenden größeren Unternehmen wie Ericsson oder Nokia auf dem Telefonsektor (Fischer/Schwarzer 1997:66). Daneben gibt es eine Reihe von Mittelständlern, die mit äußerst spezialisierten Produkten zu »Hidden Champions« wurden, also in ihrem Bereich auf dem Weltmarkt führend sind, wie ein deutscher Anbieter für elektronische Längen- und Winkelmeßsysteme, der 800 Patente entwickelt hat (Simon 1998: 63, 139). Ihre besondere Stärke besteht im allgemeinen in ihrer Nähe zu den Märkten, da sie meist intensive Kontakte zu ihren Kunden pflegen, außerdem in betrieblichen Strukturen, die oftmals das flexible Reagieren auf neue Anforderungen erlauben, da sie nicht unter bürokratischen Schwerfälligkeiten leiden.

Im übrigen zeigt das Beispiel der AEG, daß das technische Entrepreneurtum immer nur eine mögliche Option darstellte, aber von Anfang an auch andere Wege erfolgversprechend sein konnten. So konstatiert ein zeitgenössischer Beobachter um die Jahrhundertwende über diese Firma:

$»$ Mit technischer Pionierarbeit hat sie sich ... niemals aufgehalten. Sie hat neben ihren Gründungen nichts als marktgängige Waren in rationeller Massenfabrikation hergestellt und nach dem Warenhausprinzip abgesetzt. Wirtschaftlich hat sie daher das Verdienst, immer auf Verbilligung der Waren hingewirkt zu haben. Für besonders gediegene, technische Spezialausführungen hat sie dagegen niemals Sinn gehabt, und auf einen Auftrag, der mit ihren Normalien nicht zu erledigen war, hat sie meist neidlos verzichtet.«(Kreller 1903: 20)

Die starke Stellung der AEG vor 1914 beruhte offenbar auf einer anderen Basis.

\section{Dominanz durch Gründungsgeschäfte und Beteiligungen?}

Die von Rathenau 1883 gegründete Edison-Gesellschaft basierte auf der Auswertung der Edison-Patente für Glühlicht gemeinsam mit Siemens, und zwar in der Weise, daß seiner Firma die Herstellung der Glühlampen sowie Bau und Betrieb von Stromlieferungsanlagen oblag, zu denen Siemens die Maschinen lieferte. Entscheidend erwies sich in der Folge allerdings, daß die Edison-Gesellschaft sich außerdem das Recht der Vergabe von städtischen Konzessionen reserviert hatte, also neben ihrer eigentlichen elektrotechnischen Produktion ein ausgedehntes Gründungsgeschäft betrieb: Sie schuf sich ihre eigenen Abnehmer, indem sie Elektrizitätswerke begründete, die von ihr abhängig waren. Bereits vier Jahre später drängten beide Firmen auf eine Abänderung der Verträge, die insbesondere der Edison- 
Gesellschaft noch größere Bewegungsfreiheit und eine Kapitalerhöhung brachte und 1888 zur Umwandlung des Unternehmens in die »Allgemeine Elektrizitäts-Gesellschaft« führte (Kreller 1903: 11f).

Innerhalb weniger Jahre gewann die AEG mit Hilfe ihrer spezifischen Geschäftspolitik gegenüber Siemens die Oberhand. Ihre Gründungstätigkeit war so rege, daß die Methode bald als »System Rathenau« bekannt wurde:

»...die Lieferungen für fremde Besteller, das Verkaufsgeschäft, tritt zurück, und die Aufnahme der Erzeugnisse wird in der Hauptsache - auf dem Wege des Gründungsgeschäfts - von Gesellschaften besorgt, die von der Fabrikationsgesellschaft abhängig sind; in letzter Linie ist diese also ihr eigener Abnehmer und bekommt dadurch, dass sie die Emissions-Tätigkeit als Geschäftszweig mit in ihre Unternehmer-Tätigkeit einbegreift, den amphibischen Charakter des Industrie-Unternehmens und des Bankhauses.« (ebd.: 12)

In der Tat war um die Jahrhundertwende festzustellen, daß die AEG nur ein Drittel ihres Kapitals in den eigenen Produktionsmitteln investiert hatte, »während der Rest, abgesehen von Bankguthaben und Debitoren, in vollständigem oder teilweisem Besitz von anderen Unternehmungen angelegt ist, also wie bei einem Bankunternehmen vorwiegend in Effekten besteht « (ebd.: 16). Wichtige derartige Gründungen waren etwa die Berliner Elektrizitätswerke oder die Kraftübertragungs-Werke Rheinfelden.

Siemens, inzwischen unter der Leitung von Werners Sohn Wilhelm, sah sich gezwungen, zumindest teilweise auf Rathenaus Kurs einzuschwenken:

»Die Siemens-Werke folgten den Arbeiten Rathenaus in geringen Abständen und wandten sich den gleichen Gebieten und Unternehmungen zu, selten vor Rathenau, nie lange nach ihm; kein Schritt wurde ausgelassen, sie gründeten ihre Tochtergesellschaften und besondere Finanzgesellschaften, ihre Kapitalerhöhungen folgten unmittelbar denen der AEG ...«(Riedler 1916: 6).

Das Unternehmen folgte den Vorgaben seines Konkurrenten in solch einem Maß, daß die Direktion sich bei der Generalversammlung von 1901 bemüßigt fühlte, ausdrücklich zu betonen, »daß der Schwerpunkt des Hauses nach wie vor in seiner Fabrikationstätigkeit liege« (Loewe 1903: 130). Dennoch vergrößerte sich der Abstand zwischen den beiden Großfirmen in den Jahren vor dem Ersten Weltkrieg. Schon in den neunziger Jahren überflügelte die AEG mit ihrem Gesellschaftskapital wie mit ihren Beteiligungen Siemens \& Halske, auch die Dividendenzahlungen nach der Jahrhundertwende fielen wesentlich höher aus (Loewe 1903: 83, 105, Wellhöner 1989: 216.). Der sehr viel später auf den Markt getretene Newcomer brauchte nicht einmal ein Jahrzehnt, um zum tonangebenden Unternehmen der Branche zu werden und den weithin gerühmten Vorläufer auf den zweiten Platz zu verweisen.

Die Konkurrenz der zwei Konzerne hinderte sie nicht daran, sich fallweise zu Gemeinschaftsgründungen zusammenzufinden, wie etwa bei der 1890 ins Leben gerufenen Akkumulatorenfabrik Hagen i. W., die als einzige den Preiskampf der neunziger Jahre auf ihrem Markt überlebte, und der es ge- 
lang, alle übrigen 30 Unternehmen dieses Sektors zur Aufgabe zu zwingen oder aufzukaufen. Oder aber die beiden Gesellschaften einigten sich, nachdem sie mit verschiedenen technischen Systemen gegeneinander gekämpft hatten, auf die Gründung gemeinsamer Töchter wie der Gesellschaft für drahtlose Telegraphie. Ähnlich verhielt es sich bei der Entstehung von Osram, von Telefunken und der Gesellschaft für elektrische Zugbeleuchtung (Noether 1913: 71f, Blaich 1973: 177).

Ein weiteres bevorzugtes Mittel der größeren Unternehmen, um auf den verschiedenen Teilmärkten der Elektrotechnik eine Dominanz zu erringen oder zu befestigen, bestand darin, bestehende Firmen aufzukaufen oder sich an ihnen zu beteiligen. Auf diese Art entledigte sich etwa Siemens der Konkurrenzfirma Zwietusch, der deutschen Lizenzfirma der amerikanischen Western Electric Co, die dem Unternehmen auf dem Telefonsektor lange Jahren immer wieder in die Quere gekommen war: »Es wurde zunächst eine Million gezahlt und Herr Zwietusch unter unserer Leitung uns überlassen« (zit. bei Schmidt 1993a: 53).

Häufig ereignete es sich dabei allerdings, daß ein Aufkäufer nicht lange danach seinerseits aufgekauft wurde. So erwarb Schuckert \& Co 1897 die Berliner Firma Naglo; fünf Jahre später erfolgte die eingangs erwähnte Fusion der Nürnberger Schuckert-Gesellschaft mit Siemens \& Halske, wobei Siemens die Oberhand behielt. Das damals zweitgrößte Unternehmen vereinigte sich mit dem drittgrößten. Ebenfalls kurz vor der Jahrhundertwende kaufte die Helios AG die Firma Singer \& Co, hatte sich damit aber offenbar übernommen und wurde 1908 gemeinschaftlich von der AEG und Siemens erworben. Ähnlich erging es der als Zweigwerk eines amerikanischen Unternehmens fungierenden Union Elektrizitätsgesellschaft, die sich die traditionsreiche Fabrik Ludwig Loewes einverleibte, kurz nach der Jahrhundertwende jedoch ihrerseits an die AEG fiel. In diesem Fall erwarb das zweitgrößte Unternehmen das zu dieser Zeit viertgrößte. Dem gnadenlosen Prozeß von »Fressen und Gefressenwerden « entgingen nur einige wenige große Firmen, aber auch diese mußten in den Jahren vor dem Ersten Weltkrieg ihre Eigenständigkeit aufgeben: Lahmeyer fusionierte mit Felten \& Guilleaume, beide Unternehmen wurden jedoch 1910 dem Machtbereich der AEG eingegliedert; Bergmann wurde um diese Zeit von Siemens übernommen und das dritte bis dahin überlebende Unternehmen, Kummer, ging in Konkurs. Viele von denen, die in der Periode vor 1914 in Schwierigkeiten gerieten, verdankten dies im übrigen dem Umstand, daß sie sich allzusehr im Gründungsgeschäft engagiert hatten (Loewe 1903: 138ff, Kreller 1903: 26ff). Dagegen blieben dessen Vorreiter ungeschoren. Trotz des rasanten Fusionskarusells kam die von vielen erwartete - und von der Deutschen Bank vehement betriebene - Fusion der beiden Giganten AEG und Siemens weder damals noch später zustande. Auch die Verhandlungen, die 
Mitte der zwanziger Jahre und abermals während der Weltwirtschaftskrise aufgenommen wurden, blieben ergebnislos.

Seit dem Ersten Weltkrieg setzten die großen Firmen in erster Linie auf vertikale Konzentration. Die Befürchtungen, man könne Rohstoffengpässen gegenüberstehen, darüber hinaus die Hoffnung, die Qualität der Vorprodukte besser kontrollieren zu können, schließlich die Erwartung von Rationalisierungseffekten ließen sie im Krieg und verstärkt danach eigene Gießereien, Metall-, Gummi- und Preßwerke sowie Papier-, Holzwolle-, Preßspan- und Porzellanfabriken einrichten. Im Zug der Zeit lag es auch, eine stärkere Diversifizierung durch die Liaison mit Bergwerks- und Stahlhüttengesellschaften zu erreichen. Die 1920 begründete Siemens-RheinelbeSchuckert-Union hielt jedoch nur wenige Jahre. Für Siemens erwies sie sich als schlechtes Geschäft: Das hatte zu einem Teil mit den Schwierigkeiten der Ermittlung und Verteilung der Gewinne während der Inflationszeit zu tun, aber auch damit, daß diese Entscheidung im Unternehmen selbst offenbar nicht gut vorbereitet worden war. So klagte Siemens-Chef Carl Friedrich von Siemens in einem Schreiben an den Stahlindustriellen Vögeler, »daß man lange suchen kann, ehe man ein stärkeres Beispiel stärkerer Ungründlichkeit bei Abschluß eines so wichtigen und so lange dauernden Vertrages finden wird...«(zit. bei Feldenkirchen 1988: 31, FN). Ein ähnlich gelagerter Versuch der AEG, die sich seit 1921 mit anderen Großunternehmen zum AEG-Linke-Hofmann-Lauchhammer-Konzern zusammengeschlossen hatte, scheiterte ebenfalls (Pohl 1978:19, Siegrist 1980: 74).

Im Lauf der zwanziger Jahre zeigte sich überdies immer deutlicher, daß der Stern der AEG, der bis dahin so viel heller als der von Siemens geleuchtet hatte, zu sinken begann. Das ausufernde Bank- und Beteiligungsgeschäft, das vor dem Ersten Weltkrieg gerade für dieses Unternehmen äußerst profitabel gewesen war, erwies sich nach dessen Ende, in der Zeit des mäßigen und immer wieder von Rückschlägen unterbrochenen wirtschaftlichen Aufschwungs und insbesondere im Zug des konjunkturellen Niedergangs nach 1929, als Klotz am Bein. Seit Mitte der zwanziger Jahre konnte Siemens höhere Dividendenzahlungen leisten als die AEG und überflügelte den einstmals so machtvollen Rivalen inzwischen bei der Höhe des Nominalkapitals wie bei der Zahl der Beschäftigten - die Verhältnisse der Jahrhundertwende kehrten sich um (Feldenkirchen 1988: 35, FN, Siegrist 1980: 96).

Siemens gehörte zu den Unternehmen, denen die Rüstungspolitik der Nationalsozialisten neue Tätigkeitsfelder und einen sicheren Absatzmarkt bescherten. Entsprechend erreichte der Anteil des Siemens-Konzerns an den Umsätzen der deutschen Elektroindustrie nach dem Ersten Weltkrieg seinen Höchststand in den dreißiger Jahren mit 30\%. Demgegenüber wurden in der Nachkriegszeit zwar die traditionellen Geschäftsfelder der Vor- 
kriegszeit wieder aufgenommen und - beispielsweise im Kraftwerksbau oder bei der Produktion von Halbleitern - um neue ergänzt, aber gleichzeitig orientierte sich das Unternehmen mehr und mehr auch auf Gebiete jenseits der eigentlichen Elektroindustrie und erreichte niemals mehr seinen Marktanteil des Jahres 1936. 1990 belief sich dieser ebenso wie bereits 1980 auf 21\% (Czada 1969: 279, Berger 1984: 180, Berger 1993: 38).

Der häufig kolportierte Spruch, Siemens stelle heutzutage eine Bank mit angeschlossener Elektroabteilung dar, hat somit eine reale Grundlage: 1990 ebenso wie in anderen Jahren stellten die Zins- und Beteiligungserträge 2/3 des gesamten Bruttogewinns dar. »Die Finanzabteilung hat es mit einer Liquiditätsmasse von derzeit rund $20 \mathrm{Mrd}$. DM zu tun und ist eine der profitabelsten Sparten des Elektrokonzerns...« (Welzmüller 1991: 623, vgl. auch Eglau 1995: 37). 1993 gründete Siemens zur Verwaltung ihrer großen Liquiditätsreserven eine eigene Bank, die Siemens Kapitalanlagengesellschaft. Die scheinbare Novität hat ihren Vorläufer (selbst wenn dieser in einem anderen Kontext entstanden war): Ein Jahrhundert zuvor besaß Siemens neben einer anderen Finanzierungsgesellschaft die Elektrische Lichtund Anlagen-Gesellschaft in Berlin (Loewe 1903: 124, taz 23.2.1996: 6). Und ähnlich wie damals läßt die Unternehmensleitung in regelmäßigen Abständen verlauten, man plane - wie etwa 1994 in der Wirtschaftswoche berichtet wurde - eine erneute »Besinnung auf das Industriegeschäft«. Die Firma mußte einräumen, daß sie sich in vielen Bereichen durch internationale Konkurrenten zunehmend bedrängt sehe, etwa durch die französische Alcatel beim Eisenbahnbau sowie bei der Energie- und der Telekommunikationstechnik, und eine Produktivitätsoffensive plane. Deren Ziel: »Das Bankhaus Siemens soll endlich ein Industriekonzern werden« (Wirtschaftswoche Nr. 25/1994: 42ff).

Auch in neuerer Zeit hat Siemens die traditionelle Politik des Erwerbs von Beteiligungen oder auch ganzer Unternehmen fortgesetzt. Doch hat dies insgesamt nicht zu einer stetigen Ausweitung der Präsenz und Dominanz auf allen möglichen elektrotechnischen Märkten geführt, da den Einkäufen gerade in den letzten Jahren auch eine wachsende Zahl von Verkäufen gegenübersteht. So fanden Ende der achtziger Jahre unter anderem die eingangs erwähnten Übernahmen von Rolm, Arco, Teilen der britischen Plessey-Gruppe, Nixdorf und Düwag statt. Einige der neuen Töchter erwiesen sich als anhaltend hartnäckige Verlustbringer, so daß dem SiemensVorstand Heinrich v. Pierer immer öfter der größere und erfolgreichere Konkurrent und Kooperationspartner General Electric vor Augen gehalten wurde, dessen Chef Jack Welch für den Umgang mit einzelnen Bereichen nach der knappen Devise »fix, close or sell« bekannt ist. In diesem Sinn kündigte auch v. Pierer 1997 an, nachdem bereits Unternehmensteile mit einem Umsatz von 2,5 Mrd. DM abgegeben worden waren, stünden nun 
weitere mit einem Volumen von 3,5 Mrd. DM zur Disposition. Tatsächlich verkaufte das Unternehmen - mit Hinweis auf die anvisierte »Konzentration auf die Kernkompetenz« - im Oktober 1997 für 1,2 Mrd. DM ihre Rüstungselektronik an die Dasa und die British Aerospace (Berliner Zeitung, 31.10.1997). Als weitere Verkaufskandidaten im Gespräch waren die Dentaltechnik, das Leuchtengeschäft und die Kommunikationstechnik. Aus Bankkreisen war die Empfehlung zu hören, der Konzern solle sich am besten überhaupt in einzelne Gesellschaften auflösen: »... eine Aufspaltung in mehrere selbständige Einzelunternehmen könne Kräfte frei machen und den Wert für die Aktionäre erhöhen« (Lamparter 1997: 27). Viele Analysten schätzen mittlerweile »den Wert der Einzelteile höher ein als den des miteinander verwobenen Ganzen« (Fischer/Schwarzer 1997: 68). Im September dieses Jahres teilte Heinrich v. Pierer mit, man habe sich seit 1995 von Geschäftsbereichen mit einem Umsatzwert von nicht weniger als 10 Mrd. DM Umsatz getrennt, weil diese »nicht zu uns paßten, weil sie zu klein waren oder weil sie in einem anderen Verbund bessere Chancen haben« (Wirtschaftswoche Nr. 38/1998: 57).

Bemerkenswert ist der Fall der Siemens-Tochter Nixdorf (SNI), deren geplante Übernahme durch den taiwanischen Acer-Konzern bisher durch die asiatische Finanzkrise vereitelt wurde. Das Unternehmen hatte der Muttergesellschaft seit der Übernahme einen Verlust von mehr als 2 Mrd. DM beschert, der unter anderem auf die Beteiligungen der Tochter an einer PCHandelskette und an einem US-amerikanischen Produzenten zurückging: »Übernahmen sind nicht nur eine Frage des Geldes, sondern vor allem eine Frage des Zusammenpassens « resümierte der SNI-Chef Schulmeyer (Fischer/Schwarzer 1997: 76) Auch hier handelte es sich - man erinnere sich an die fatalen Ergebnisse des Fusionsfiebers um die Jahrhundertwende um eine Kette von Übernahmen, die mehreren der daran beteiligten Firmen erhebliche Verluste statt des erhofften geldwerten Nutzens im Interesse der Shareholder brachten.

Noch wechselhafter gestalteten sich die Geschicke der AEG in der Nachkriegszeit. Auch sie hatte vom Rüstungsboom des nationalsozialistischen Regimes profitiert und verfügte in den Jahren des Wirtschaftswunders nach wie vor über umfangreiche Beteiligungen, unter anderem an Osram, an der Kraftwerk-Union sowie an Hartmann \& Braun. Wie der mächtigere Konkurrent Siemens gliederte sie sich darüber hinaus weitere Unternehmen an, etwa die Bahntechnik von MBB und MAN oder die Bürotechnik von Olympia. Doch seit den siebziger Jahren zeigten sich immer deutlichere Zeichen des wirtschaftlichen Niedergangs. Im Jahr 1974 konnte bereits keine Dividende mehr gezahlt werden und die Branchen-Kenner diagnostizierten gravierende Management-Probleme, eine wachsende Verschuldung und den Verlust der Konkurrenzfähigkeit auf wichtigen Märkten, unter anderem 
bei Haushaltsgeräten. So wurde das Unternehmen, das bis dahin so viele andere auf kaltem Wege liquidiert hatte, 1985 schließlich selbst von einem größeren Unternehmen übernommen, dem Daimler-Benz-Konzern - eine Transaktion, die diesem in den nächsten Jahren Verluste von 5 Mrd. DM bescherte. Die AEG war mittlerweile zu einem »Sammelsurium von Firmen, die wenig miteinander zu tun hatten«, geworden und trug nun ihrerseits dazu bei, die neue Mutterfirma, die sich mit der vagen Hoffnung auf "Synergieeffekte « ein buntes Spektrum von Unternehmen zusammengekauft hatte, in Schwierigkeiten zu bringen (Eglau 1995: 37).

\section{Dominanz durch Kooperationen und Kartellbildungen?}

Die Entwicklung der Elektroindustrie ist seit jeher nicht nur durch Gründungsgeschäfte und Beteiligungen, sondern auch durch vielfältige Kooperationen gekennzeichnet. Bereits die Gründung des AEG-Vorläufers, der Edison-Gesellschaft, ging, wie weiter oben beschrieben, auf eine derartige Kooperation zurück. Solche und andere Formen der wechselseitigen Abstimmung von großen Firmen in mehr oder weniger verbindlichen Formen sollten nicht nur die Zeit vor dem Ersten Weltkrieg, sondern auch noch spätere Perioden prägen. Erste Ansätze hierzu gab es bereits Ende der siebziger Jahre, als Siemens auf ausdrücklichen Wunsch des Generalpostmeisters Stephan bei der Lieferung von Guttapercha-Kabeln für den TelegrafenDienst der Reichspost die Zusammenarbeit mit Felten \& Guilleaume aufnahm, so daß die entsprechenden Aufträge in der Folge teils gemeinsam erfüllt, teils regional aufgeteilt wurden. Preisabsprachen gab es daneben auch bei militärischen Lieferungen von Armierungskabeln für Fortifikationen, von Feldkabeln und bei solchen für den Bedarf des Landheeres (Schmidt 1993a: 50).

Gegen Ende des Jahrhunderts nahm die auch bisher bereits übliche Preiskonkurrenz auf den meisten Märkten der Elektroindustrie angesichts der bestehenden Überkapazitäten weiter zu. Dies veranlaßte die davon betroffenen Unternehmen, nach Abkommen Ausschau zu halten, wie sie ihnen in den davorliegenden Jahren von zahlreichen Rohstoffproduzenten mit Erfolg vorgeführt worden waren. Insbesondere gelang es Kohlezechen und Kupferhütten, ihre Absatzmärkte zu regulieren und die Preise heraufzusetzen. Entsprechend verständigte sich Siemens beispielsweise mit der AEG über die »Regelung einer gemeinschaftlichen Behandlung der größeren selbständigen elektrischen Unternehmungen in Südamerika«. Auch schlossen sich kurz vor der Jahrhundertwende unter Führung von Siemens \& Halske die größeren Unternehmen der Branche in loser Form zusammen, um Preissteigerungen für bestimmte Fertigfabrikate zu erreichen. Man einigte sich darauf, daß die beteiligten Firmen ihre Listenpreise für Dyna- 
momaschinen, Motoren, Transformatoren, Regulier- und Anlaßapparate um $5 \%$ erhöhen sollten, doch wurden damit nur vorübergehend »bessere Preise« erzielt, da viele Unternehmen es mit dem konjunkturellen Einbruch um die Jahrhundertwende vorzogen, die frühere Gepflogenheit der Preisunterbietung wieder aufzunehmen. Wirksamer erwies sich demgegenüber das um 1902 begründete Geheimkartell zwischen der AEG, Felten \& GuilleaumeLahmeyer und Siemens-Schuckert, das auf einen »Submissionsschutz« bei Starkstromgeschäften mit staatlichen Behörden oder städtischen Gemeinden abzielte, indem die drei Firmen sich gegenseitig verpflichteten, bei größeren Projekten jeweils einer der drei Vertragsfirmen die Ausführung der Installationsarbeiten zu überlassen. Demgegenüber konnten die kleineren Installationsfirmen, die hiermit ausgebootet werden sollten, einzig den Vorteil der Kundennähe geltend machen (Schmidt 1993a: 51, Mayr 1922: 46f, Blaich 1973: 177).

Eine ebenfalls nur wenige Jahre lang erfolgreiche Absprache war die 1903 von der AEG initiierte Verkaufsstelle Vereinigter Glühlampenfabriken $(V V G)$, in der sich fast sämtliche Glühlampenfabriken Deutschlands, Österreich-Ungarns und Hollands zusammenschlossen, die aber durch die Entwicklung neuartiger Metallfadenlampen obsolet wurde. Sie fand nach dem Ersten Weltkrieg ihre Fortsetzung in Form des Phoebus-Vertrages, eines weltweiten Kartells, das neben Osram und der International Electric Company auch Philips und Tungsram sowie die wichtigsten Unternehmen Englands, Italiens, Frankreichs und der Schweiz umfaßte. Das Abkommen legte weltweit maximale Gebietsquoten fest und regelte den internen Austausch von Patenten sowie die Vergabe von Lizenzen, vor allem aber einigte man sich zum Zweck der Absatzsteigerung darauf, die Lebensdauer der Lampen auf 1.000 Stunden zu begrenzen. Es handelte sich in der Folge um einen der seltenen Fälle eines tatsächlich über längere Zeit funktionierenden Abkommens, das nahezu den ganzen Globus umfaßte. Dies unterschied diese Sparte von sonstigen Bereichen des Starkstromsektors, bei denen vor 1914 ähnlich wirksame, aber sehr viel weniger weitreichende Kartell-Regelungen lediglich für Dampfturbinen zustandekamen (vgl. Schmidt 1993b: 43, 83ff).

Auch im Bereich des Schwachstroms sahen die Verhältnisse im einzelnen höchst unterschiedlich aus. Erst kurz vor dem Ersten Weltkrieg trafen die führenden deutschen Firmen Abmachungen über einen zehnprozentigen Preisaufschlag bei Telefonen. Um diese Zeit erfolgte auch, wiederum mit Zustimmung und Förderung der Reichspost, eine Neuauflage des Kabelkartells der siebziger Jahre. Der Deutsche Schwachstromkabel-Verband umfaßte diesmal fünfzehn Unternehmen und somit direkt oder indirekt sämtliche deutschen Kabelhersteller, die sich untereinander auf Preisabsprachen und Absatzkontingente einigten. Diese Vereinigung bestand bis 1945, wies 
den beteiligten Unternehmen in Absprache mit der Reichspost Quoten zu und erreichte zumindest gegenüber dieser, ihr Interesse an »zeitgemäßen Preisaufschlägen « (wie es in einem internen Siemens-Bericht heißt) durchzusetzen. Ansonsten zeichnete sie sich aber keineswegs durch besondere Effektivität aus, genausowenig wie sie Streitigkeiten und Prozesse der Partnerfirmen um Patente ausschloß (Wessel 1982: 22ff, Schmidt 1993b: 109ff).

Bereits vor dem Ersten Weltkrieg entstanden weitere internationaler Absprachen, so wenn Siemens 1913 mit der in den USA ansässigen Western Electric Co. einen Vertrag abschloß, der den gegenseitigen Austausch von Patenten wie auch von Erfahrungen mit Produktentwicklungen und Organisationsfragen, insbesondere aber die »Verteilung des Weltgeschäftes« zum Inhalt hatte. Eine ähnliche Vereinbarung gab es auf dem Gebiet der drahtlosen Telegraphie zwischen der Londoner Wireless Telegraph Co. von Marconi und der deutschen Telefunkengesellschaft. Vor allem die Weltwirtschaftskrise stellte einen weiteren Anstoß dar, auf internationaler Ebene Interessengemeinschaften zu bilden. So wurde in einem Vertrag zwischen Siemens und Westinghouse für mehrere Sparten der Austausch von Patenten und Know How ebenso wie die Abgrenzung von Interessensgebieten festgelegt. Ähnliche Abmachungen existierten mit der General Electric Company und mit der schweizerischen BBC sowie auf dem Telefonmarkt mit der amerikanischen ATT und der britischen IAT. Aber auch im Ausland gestalteten sich die Verhältnisse der kooperierenden Firmen nicht immer harmonisch, sondern ähnlich wie im Inland als Nebeneinander von $\mathrm{Zu}-$ sammenarbeit und heftigen Kämpfen. Beispielsweise beschuldigten sich Siemens und ihre englischen wie US-amerikanischen Vertragspartner in der Nachkriegszeit wechselseitig, neben ihren offiziellen Verträgen auch noch solche geheimer Art getroffen zu haben. Die Kontrahenten riefen schließlich ein Schiedsgericht in London an (Schmidt 1993b: 53, Die elektrotechnische Industrie der Welt 1927: 677, Feldenkirchen 1988: 52).

Der Omgus-Bericht zu Siemens geht für die Zeit des Nationalsozialismus davon aus, Siemens sei an etwa 1.000 Kartellen beteiligt gewesen, dagegen verzeichnet das deutsche Kartellverzeichnis von 1935 in der Elektroindustrie lediglich 67 Kartelle (Feldenkirchen 1988: 52). In jedem Fall ist zu berücksichtigen, daß die formale Existenz von Kartellen nicht allzuviel über deren Wirksamkeit aussagt. Führende Vertreter der Elektrofirmen äußerten sich in internen Stellungnahmen wiederholt skeptisch in bezug auf ihren Nutzen, so etwa ein AEG-Manager 1930 gegenüber Carl Friedrich von Siemens:

»Vom rein geschäftlichen Standpunkt aus glauben wir die Erfahrung gemacht zu haben, dass Kartelle wenn überhaupt, nur auf klar abgegrenzten Einzelgebieten für verhältnismäßig einfache Konstruktionen erfolgversprechend, nicht dagegen zur Regelung der Zusammenarbeit 
weit verzweigter großer Industrien geeignet sind. Aber auch auf eng begrenzten Gebieten sind die Erfahrungen meist nur vorübergehend gut, à la longue ergeben sich auch hier grosse Schwierigkeiten, wie das Glühlampenkartell, das Zählerkartell und die Kabelkartelle zeigen. Ganz zu schweigen von den loseren Bindungen« (zit. bei Feldenkirchen 1988: 54, FN).

Diese Fragwürdigkeit der Vorteile von Kartellen für die daran beteiligten Firmen dürfte während des Nationalsozialismus noch deutlicher geworden sein. In dieser Zeit stieg zwar die Zahl der Kartelle - auch in der Elektroindustrie - an, gleichzeitig verloren aber zumindest Preisabsprachen zunehmend an Bedeutung, da mit einer Ausweitung des Systems von Kontingentierungen und Bezugsscheinen andere Dimensionen (etwa die Beziehungen zur Partei- und Staatsbürokratie) wichtiger wurden (Pohl 1978: 31ff). Darüber hinaus lag ein wichtiger Grund für den immer wieder begrenzten Erfolg der Kartellierungsbestrebungen in der Dynamik der technischen Entwicklung, da Abkommen sich immer auf bereits existierende und standardisierte Produkte beziehen konnten, deren Bedeutung jedoch möglicherweise bald durch Innovationen in Frage gestellt wurde. Außerdem waren solche relativ lockeren Verbindungen immer wieder dadurch gefährdet, daß die beteiligten Firmen bei sich ändernden Marktbedingungen ihr Interesse eher in Alleingängen als in der Einhaltung von Absprachen sahen.

In der Zeit nach dem Zweiten Weltkrieg setzte das grundsätzliche Verbot von Kartellen in der deutschen Wettbewerbsgesetzgebung im Vergleich zu den früher äußerst kartellfreundlichen Regelungen grundlegend andere Ausgangsbedingungen. Als Ersatz für diese einstmals so verbreitete Form haben sich in den letzten Jahrzehnten Unternehmens-Netzwerke herausgebildet, von denen behauptet wird, sie repräsentierten »ein modernisiertes Regime der regulierten Konkurrenz« (Windolf/Beyer 1995: 3). Die großen Firmen sind in der Tat eine Reihe von nationalen und internationalen Allianzen und joint ventures eingegangen - beispielsweise Siemens seit den achtziger Jahren solche für die gemeinsame Entwicklung und den Vertrieb von Mikrochips (mit IBM), von Halbleitern, Medizintechnik, Lampen und integrierten Schaltungen (mit verschiedenen japanischen Firmen), von Druckwasser-Atomreaktoren (mit der französischen Firma Framatom), von Solarzellen, Leistungshalbleitern, Supraleitern und Kernkraft-Hochtemperaturreaktoren (mit verschiedenen in Deutschland ansässigen Firmen) (Welzmüller 1991: 623). Ähnlich wie bei den früher oft konflikthaften Beziehungen zu Kartellpartnern gehen auch in der Gegenwart Kooperationen mit Rechtsstreitigkeiten Hand in Hand - so arbeiteten in den letzten Jahren Siemens und die Alcatel-Tochter GEC-Alsthom nicht nur an mehreren gemeinsamen Projekten im Verkehrsbereich, sondern die beiden Firmen trafen sich wegen eines südkoreanischen Auftrags für Hochgeschwindigkeitszüge, der an die französische Firma ging, auch vor Gericht (Wirtschaftswoche Nr. 25/1994: 44). 


\section{Fazit}

Im Hinblick auf die eingangs angeführten Prophezeiungen über den unaufhaltsamen Siegeszug der großen Konzerne in der Elektroindustrie ist das Ergebnis des historischen Rückblicks ein zwiespältiges. Frühe Gründungen wie Siemens oder die AEG konnten sich tatsächlich über Jahrzehnte hinweg eine besondere Position sichern, und dies mit recht unterschiedlichen Strategien. Keine davon erwies sich gleichwohl als Königsweg, sondern alle waren je nach Situation und Kontext mehr - oder auch weniger von geschäftlichem Erfolg gekrönt. Ein einmal eingenommener Vorsprung bei Forschung und Entwicklung mußte ebensowenig eine dauerhafte Überlegenheit in diesem Bereich gewährleisten, wie dieses Mittel stets unabdingbar war, um sich auf dem Markt durchzusetzen. Ebenso konnte die Beteiligung an anderen Unternehmen, ob in der Form von »Elefantenhochzeiten « oder von Aufkäufen kleinerer Firmen, die Position des Aufkäufers genausogut verbessern wie endgültig unterminieren. Die heutzutage als besonders neu geltenden internationalen Kooperationen gab es in der Elektroindustrie schließlich bereits vor dem Ersten Weltkrieg, wenngleich in kleineren Dimensionen. Sie konnten synergetisch wirksam werden oder aber in kostspieligen Auseinandersetzungen enden.

Die Dynamik des Wettbewerbs zeigt sich nicht nur in der unterschiedlichen Bedeutung solcher Strategien in einzelnen Perioden, sondern auch in den diversen Wellen von späteren Neugründungen sowie schließlich daran, daß einer der beiden Großen mittlerweile als eigenständiges Unternehmen überhaupt von der Bildfläche verschwunden ist und damit demonstriert, wie sehr selbst derartige Giganten dem harten Wind der kapitalistischen Konkurrenz ausgesetzt bleiben.

Das Schicksal des überlebenden Rivalen scheint hingegen doch eher für die Vermutung der »geschlossenen Gesellschaft« zu sprechen. Tatsächlich zeigt sich ganz allgemein in der deutschen Wirtschaft, daß für alle Gründungen nach dem Ersten Weltkrieg der Weg in die Spitzenpositionen länger dauerte und schwieriger wurde als für die früheren. So ist es etwa nur einem einzigen nach 1945 gegründeten Unternehmen gelungen, in den folgenden beiden Jahrzehnten in den Kreis der hundert Größten aufzusteigen: Grundig. Siemens hingegen gehört (neben der Deutschen Bank) ununterbrochen zu den zehn größten AGs des Deutschen Reiches bzw. der Bundesrepublik. Auch eine Analyse der Personal- und Kapitalverflechtungen von 1976 belegt, daß der Konzern eine der fünfzehn zentralen Unternehmensgruppen in der Bundesrepublik darstellt, mit Verbindungen zu den beiden Hauptzentren Dresdner Bank und Deutsche Bank. Eine ähnliche Untersuchung der 623 größten Unternehmen von 1992 identifiziert Sie- 
mens neben der Deutschen Bank, der Allianz und Daimler-Benz als eines der »Zentren hierarchischer Kontrolle« (Weder 1969: 40, 152, Pappi u.a. 1987: 712f, Windolf/Beyer 1995: 12). Eine Machtstellung, die das Unternehmen unter anderem dazu nutzen konnte, um einen besonders erfolgreichen Lobbyismus zu praktizieren, in den letzten Jahrzehnten beispielsweise bei der Akquisition von Subventionen für vermeintlich zukunftsweisende technologische Großprojekte wie Atomtechnologie, Eurofighter oder Transrapid.

Und doch: Das Bild des machtvollen Siemens-Imperiums hat gerade in den letzten Jahren erheblich an Glanz eingebüßt. In der neueren Berichterstattung von Manager-Magazinen blickt man keineswegs mehr voll ehrfürchtiger Bewunderung auf den Konzern, sondern gefällt sich eher darin, InsiderEinschätzungen zu kolportieren, wonach sich dieser durch Risikovermeidung und eine Kultur des Mißtrauens auszeichnen soll. Tatsächlich sah sich die Konzernspitze jüngst zu Reorganisationen veranlaßt, bei denen ausgerechnet jene Form von Unternehmen als Modell präsentiert werden, die lange Zeit als besonders altbacken gegolten hatte. So verkündete Heinrich v. Pierer 1996 in einem Interview: »Ein großes Unternehmen wie Siemens muß dezentral organisiert sein. Dem haben wir durch unsere Unternehmensreform Rechnung getragen. Wir haben 250 Geschäftseinheiten geschaffen, die wie mittelständische Firmen eigenständig am Markt agieren können. Wir nennen sie unsere 'Schnellboote'« (Wirtschaftswoche Nr. 20/1996: 120). Über den Erfolg dieser erneuten Produktivitätsoffensive sind die Meinungen noch geteilt, doch für den Großteil der 573.000 Aktionäre - davon fast $1 / 3$ Investmentgesellschaften - erscheint es ohnehin ziemlich belanglos, mit welchen Mitteln die Ergebnisse verbessert werden, und was dabei aus den weltweit rd. 400.000 Arbeitsplätzen wird. Ihnen geht es einzig und allein um den Wert der Siemens-Aktien im Vergleich zum Dax. Dieser aber hinkt seit Beginn der neunziger Jahre der allgemeinen Entwicklung hinterher, und so muß sich der Chef des größten deutschen Elektro-Konzerns im Herbst 1998 von Wirtschaftsjournalisten fragen lassen: »Herr v. Pierer, ihre Gewinnprognose haben Sie verfehlt, aus einigen Geschäftsfeldern kommen nur noch Hiobsbotschaften - macht Ihnen der Job noch Spaß?« (Wirtschaftswoche Nr. 38/1998: 56).

\section{Literatur}

Arbeitsgruppe Alternative Wirtschaftspolitik (1988): Wirtschaftsmacht in der Marktwirtschaft - Zur ökonomischen Konzentration in der Bundesrepublik, Köln.

Bähr, Johannes (1997): Substanzverluste, Wiederaufbau und Strukturveränderungen in der deutschen Elektroindustrie 1945-1955, in: Die elektrotechnische Industrie nach 1945 (Geschichte der Elektrotechnik 15, T. 1), Berlin-Offenbach: 61ff.

Berger, Manfred (1984): Elektrotechnische Industrie, Berlin-München.

Berger, Manfred (1993): Elektroindustrie, Berlin-München. 
Blaich, Fritz (1973): Kartell- und Monopolpolitik im kaiserlichen Deutschland, Düsseldorf. Breitenacher, Michael u.a. (1974): Elektrotechnische Industrie, Berlin-München.

Peter Czada (1969): Die Berliner Elektroindustrie in der Weimarer Zeit, Berlin.

Cramer, Ulrich (1987): Klein- und Mittelbetriebe: Hoffnungsträger der Beschäftigungspolitik? in: Mitteilungen des Instituts für Arbeitsmarkt- und Berufsforschung, H. 1: $15 \mathrm{ff}$.

Die elektrotechnische Industrie der Welt (1927), in: Elektrotechnische Zeitschrift, H. 20: $675 f f$.

Eglau, Hans Otto (1997): Ende eines Trauerspiels, in: Die Zeit, Nr. 46: 37f.

Fischer, Gabriele; Schwarzer, Ursula (1997): Die ewigen Verfolger, in: Manager-Magazin April: 59ff.

Fischer, Wolfram (1976): Bergbau, Industrie und Handwerk 1914-1970, in: Aubin, Hermann; Zorn, Wolfgang (Hrsg.): Handbuch der deutschen Wirtschafts- und Sozialgeschichte, Bd. 2, Stuttgart: $796 \mathrm{ff}$.

Germann, Friedrich (1940): Stellung der Spezialfabriken, des Handwerks und des Großhandels in der Elektroindustrie, Diss., Berlin .

Hilbert, Josef; Sperling, Hans Joachim (1990): Die kleine Fabrik, München-Mering.

Kreller, Emil (1903): Die Entwicklung der deutschen elektrotechnischen Industrie und ihre Aussichten auf dem Weltmarkt, Leipzig.

Lamparter, Dietmar H. (1997): Von Menschen und Märkten, in: Die Zeit, Nr. 25: 27f

Leicht, René (1995): Die Prosperität kleiner Betriebe, Heidelberg.

Lenin, W. I. (1917): Der Imperialismus als höchstes Stadium des Kapitalismus, Berlin, 1979.

Loewe, Josef (1903): Die elektrotechnische Industrie, in: Schriften des Vereins für Socialpolitik, N. 107, Leipzig, 77ff.

Marx, Karl (1867): Das Kapital, Bd. 1, MEW 23, Berlin.

Matschoß, Conrad (Hrsg.) (1916): Werner Siemens - Ein kurzgefaßtes Lebensbild nebst einer Auswahl seiner Briefe, Bd. 2, Berlin.

Mayr, Otto (1922): Wirtschaftskampf zwischen elektrotechnischen Installationsfirmen und Elektrizitätswerken bzw. -Gesellschaften und Stellungnahme der deutschen Regierungsstellen hierzu, Diss., Erlangen.

Müller; Helene (1925): Der Konkurrenzkampf zwischen Elektro-Großindustrie und Kleingewerbe, Diss., Ffm.

Naschold, Frieder (1997): Die Siemens AG: Inkrementale Anpassung oder Unternehmenstransformation? - Eine Fallstudie über Kontinuität und Wandel eines Konzerns- WZB discussion papers FS II 97-201, Berlin.

Noether, Erich (1913): Vertrustung und Monopolfrage in der deutschen Elektrizitätsindustrie, Diss., Heidelberg.

Pappi, Franz Urban u.a. (1987): Die Struktur der Unternehmensverflechtungen in der Bundesrepublik, in: Kölner Zeitschrift für Soziologie und Sozialpsychologie: 669ff.

Petzold, Hartmut (1987): Zur Entstehung der elektronischen Technologie in Deutschland und in den USA, in: Geschichte und Gesellschaft, Bd. 13/1987: 340ff.

Pohl, Hans (1978): Die Konzentration in der deutschen Wirtschaft vom ausgehenden 19. Jahrhundert bis 1945, in: Die Konzentration in der deutschen W irtschaft seit dem 19. Jahrhundert, Zeitschrift für Unternehmensgeschichte, Beiheft 11, Wiesbaden: 4ff.

Riedler, Alois (1916): Emil Rathenau und das Werden der Großindustrie, Berlin.

Rogge, Henning (1983): Fabrikwelt um die Jahrhundertwende am Beispiel der AEG Maschinenfabrik in Berlin-Wedding, Köln.

Schmidt, Dorothea (1993a): Massenhafte Produktion - Produkte, Produktion und Beschäftigte im Stammwerk von Siemens vor 1914, Münster.

Schmidt, Dorothea (1993b): Weder Ford noch Taylor - Zu Rhetorik und Praxis der Rationalisierung in den zwanziger Jahren am Beispiel dreier Siemens-Werke, Bremen.

Siegel, Tilla; von Freyberg, Thomas (1991): Industrielle Rationalisierung unter dem Nationalsozialismus, Ffm., New York.

Siegrist, Hannes (1980): Deutsche Großunternehmen vom späten 19. Jahrhundert bis zur Weimarer Republik, in: Geschichte und Gesellschaft: $60 \mathrm{ff}$.

Simon, Hermann (1998): Die heimlichen Gewinner - »Hidden Champions«, München. 
Sombart, Werner (1928): Der moderne Kapitalismus Bd. III/2: Das Wirtschaftsleben im Zeitalter des Hochkapitalismus, München-Leipzig.

Voigt, Heinrich (1925): Nachdenkliches und Heiteres aus den ersten Jahrzehnten der Elektrotechnik, Leipzig.

Weder, Dietrich (1969): Die 200 größten deutschen Aktiengesellschaften 1913-1962, Diss. Ffm.

Wellhöner, Volker (1989): Großbanken und Großindustrie im Kaiserreich, Göttingen.

Welzmüller, Rudolf (1991): Siemens - Eine Unternehmensanalyse, in: WSI-Mitteilungen 10: 620ff.

Welzmüller, Rudolf (1992): Branchenanalyse Elektro-, Elektronik- und EDV-Industrie, Ffm.

Wessel, Horst A. (1982): Der deutsche Schwachstromkabelverband, in: Zeitschrift für Unternehmensgeschichte, Bd. 27: 22ff.

Windolf, Paul; Beyer, Jürgen (1995): Kooperativer Kapitalismus - Unternehmensverflechtungen im internationalen Vergleich, in: Kölner Zeitschrift für Soziologie und Sozialpsychologie, H. 1: 1ff.

Wittke, Volker (1996): Wie entstand industrielle Massenproduktion? Berlin.

Zettler, Alois (o.J.): Notizen und Gedanken zur Elektrotechnik-Firmenschrift der Fa. Alois Zettler, o.O.. 